\title{
THE SINGLET SYSTEM OF THE OXYGEN ARC SPECTRUM AND THE ORIGIN OF THE GREEN AURORAL LINE
}

\author{
BY RUdOLF FreRichs* \\ California Institute of Technology, Pasadena
}

(Received June 2, 1930)

\section{Abstract}

The oxygen arc spectrum has been investigated under varied conditions in the extreme ultraviolet. A number of observed lines have been arranged in the singlet system of terms. The values of the low terms ${ }^{1} D_{2}$ and ${ }^{1} S_{0}$ have been fixed

\begin{abstract}
A S J. C. McLennan has shown, ${ }^{1}$ the green auroral line $\lambda=5577.35 \mathrm{~A}$ gives the Zeeman pattern of a singlet line and is therefore probably the forbidden combination between the low metastable terms ${ }^{1} D_{2}$ and ${ }^{1} S_{0}$ of the oxygen atom. Although many attempts have been made to locate these terms, either by extrapolating the values of the corresponding terms of similar spectra or by connecting the structure of the oxygen band spectra with the dissociation energy, the exact position of these terms could not be fixed. The direct method of locating the terms by spectroscopic analysis was not used in former investigations.

The following paper gives an attempt to classify all the stronger ultraviolet singlet lines of the oxygen atom. The classification of singlet lines is usually more difficult than the classification of lines of higher multiplicity. Therefore, we have investigated the oxygen arc spectrum under different controlled conditions in order to obtain information regarding the excitation potential of the different lines. The results so obtained are given in part 2 after a short description of the experimental arrangement in part 1. Part 3 discusses the series analysis of the new lines.
\end{abstract}

\section{Apparatus and Procedure}

Two different gratings were used. The first one, ruled by Anderson, (Focus $=1 \mathrm{~m}$, area $5 \times 8 \mathrm{~cm}$, dispersion : first order $17 \mathrm{~A}$ per $\mathrm{mm}$ ) gives very good definition (see Table I) but unfortunately scatters the light so much that it could not be used for weak lines. The other one, ruled at the National Physical Laboratory, (Focus $=1 \mathrm{~m}$, area $3.5 \times 5 \mathrm{~cm}$, dispersion $18 \mathrm{~A}$ per $\mathrm{mm}$ ) shows very bright and sharp lines down to 520A, the smallest wave-length here investigated, although it was not made with light rulings for the ultraviolet.

The spectrograph built for this investigation was very simply constructed. It consists of a brass tube which carries at one end the grating, at the other

* International Education Board Fellow.

${ }^{1}$ J. C. McLennan, J. H. McLeod, and McQuarrie, Proc. Roy. Soc. A114, 1 (1927). J. C. McLennan, J. H. McLeod and R. Ruedy, Phil. Mag. 6, 558 (1928);also L. A. Sommer, Zeits. f. Physik 51, 451 (1928) 
end, the slit and holder for uncurved plates $1^{\prime \prime} \times 4^{\prime \prime}$ in size. The cover plates on the two ends of the spectrograph are sealed with rubber gaskets inserted in small circular grooves and are held in place with screws. These gaskets proved to be very satisfactory. Over one hundred exposures were made with out the slightest trouble from leaks in the spectrograph.

Much care was taken in the arrangement of the pumping system and the light source. Small traces of oxygen in the spectrograph cause absorption of the light as well as serious fogging of the plates. In order to obtain as great a pressure difference as possible between the discharge tube and spectrograph we used two slits in series. The outer slit, narrow $(0.01 \mathrm{~mm})$ and short $(2 \mathrm{~mm})$ was sealed to the spectrograph so that the only communication between the discharge tube and the spectrograph was through this narrow aperture. A small distance behind this slit, there was a second slightly larger slit arranged at the end of a conical tube. One two-stage pump evacuated the space between these two slits, a second one the spectrograph directly, all connections were made with short and large tubes.

In order to make experiments on the excitation energy of the singlet lines the spectrograph was equipped with arrangements to work either with pure oxygen or with circulating mixtures of oxygen and rare gases. The oxygen was generated electrolytically. Small traces of hydrogen could be oxidized by a glowing platinum wire. The gas was dried with phosphorus pentoxide and then stored under low pressure in a bulb with a side tube immersed in liquid air. The flow of the oxygen in the discharge tube was regulated by a narrow capillary. A variable valve was not used but the amount of gas flowing in the tube was kept exactly constant by regulating the pressure in the storage bulb. The discharge tube was evacuated through two single stage pumps in series. For the experiments with pure oxygen, these four pumps were backed by an oil-pump. For the experiments with helium-oxygen and with neon-oxygen, the four pumps exhausted the gas from the spectrograph and discharge tube in to a large bulb with a back pressure of about five millimeters. From this bulb the gas flowed back to the discharge tube through a heated quartz tube with copper oxide and a large charcoal trap in liquid air. The charcoal kept all impurities back and after a short time of circulation, the spectrum of the discharge tube was free from impurities. The charcoal also adsorbed the oxygen of the mixture and we had therefore to add continuously a small amount of oxygen to the rare gas after it had passed the charcoal. Argon is strongly adsorbed by charcoal. Therefore, the argonoxygen mixture was circulated over calcium heated to about 300 degrees $\mathrm{C}$ in an iron tube and through a trap in liquid air, and new oxygen was added to replace that absorbed in the calcium tube.

The experiments were made with the positive column as well as with the hollow cathode in pure oxygen and in different mixtures. The discharge tube that was finally adopted had a diameter of about $15 \mathrm{~mm}$ and a length of $250 \mathrm{~mm}$. Large electrodes of heavy aluminum allowed the use of currents of between 350 and 750 milliamperes. Direct current at 1300 volts was supplied by two small generators of 650 volts each, connected in series. Suit- 
able ballast-resistance was used. The hollow cathode consisted either of a small aluminum tube $10 \mathrm{~mm}$ in diameter and $40 \mathrm{~mm}$ in length or of a hole of similar size in a solid piece of aluminum, which was cooled by water flowing through a copper-tubing surrounding the aluminum. The hollow cathodes carried currents of between 150 and 500 milliamperes.

TABLE I. Ultraviolet OI lines.

\begin{tabular}{|c|c|c|c|c|c|}
\hline Intensity & Wave-length & $\begin{array}{l}\text { Frequency } \\
\text { obs. }\end{array}$ & $\begin{array}{l}\text { Frequency } \\
\text { calc. }\end{array}$ & Classification & \\
\hline $\begin{array}{l}1 \\
3 \\
3 \\
4 \\
3 \\
1\end{array}$ & $\begin{array}{r}1217.60 \\
1152.06 \\
999.47 \\
990.86 \\
990.27 \\
990.21 \\
988.84 \\
988.72 \\
988.66 \\
935.15 \\
922.02 \\
882.88 \\
879.65 \\
879.14 \\
877.97\end{array}$ & $\begin{array}{r}82128 \\
86801 \\
100053 \\
100922 \\
100983 \\
100989 \\
101128 \\
101141 \\
101147 \\
106935 \\
108457 \\
113266 \\
113681 \\
113747 \\
\\
113899\end{array}$ & $\begin{array}{r}82128 \\
86801 \\
100053 \\
100922 \\
100983 \\
100989 \\
101128 \\
101141 \\
101147 \\
106934 \\
108457 \\
113265 \\
113687 \\
\{113744 \\
113755 \\
113761 \\
113903 \\
113914\end{array}$ & $\begin{array}{l}2 p^{1} S_{0}-\left({ }^{2} P\right) 3 s^{1} P_{1} \\
2 p^{1} D_{2}-\left({ }^{2} D\right) 3 s^{1} D_{2} \\
2 p^{1} D_{2}-\left({ }^{2} P\right) 3 s^{1} P_{1} \\
2 p^{3} P_{0}-\left({ }^{2} D\right) 3 s^{3} D_{1} \\
2 p^{3} P_{1}-\left({ }^{2} D\right) 3 s^{3} D_{2} \\
2 p^{3} P_{1}-\left({ }^{2} D\right) 3 s^{3} D_{1} \\
2 p^{3} P_{2}-\left({ }^{2} D\right) 3 s^{3} D_{3} \\
3 p^{3} P_{2}-\left({ }^{2} D\right) 3 s^{3} D_{2} \\
2 p^{3} P_{2}-\left({ }^{2} D\right) 3 s^{3} D_{1} \\
2 p^{1} D_{2}-(2 D) 4 s^{1} D_{2} \\
2 p^{1} D_{2}-(2 D) 3 d^{1} F_{3} \\
2 p^{1} D_{2}-(2 D) 5 s^{1} D_{2} \\
2 p^{3} P_{0}-\left({ }^{2} P\right) 3 s^{3} P_{1} \\
2 p^{3} P_{1}-\left({ }^{2} P\right) 3 s^{3} P_{2} \\
2 p^{3} P_{1}-\left({ }^{2} P\right) 3 s^{3} P_{1} \\
2 p^{3} P_{1}-\left({ }^{2} P\right) 3 s^{3} P_{0} \\
2 p^{3} P_{2}-(2 P) 3 s^{3} P_{2} \\
2 p^{3} P_{2}-\left({ }^{2} P\right) 3 s^{3} P_{1}\end{array}$ & II. Order \\
\hline & $\begin{array}{l}861.63 \\
850.74\end{array}$ & $\begin{array}{l}116059 \\
117545\end{array}$ & $\begin{array}{l}116058 \\
117544\end{array}$ & $\begin{array}{l}2 p^{1} D_{2}-\left({ }^{2} D\right) 6 s^{1} D_{2} \\
2 p^{1} D_{2}-\left({ }^{2} D\right) 7 s^{1} D_{2}\end{array}$ & I.Order \\
\hline $\begin{array}{l}3 \\
1 \\
1 \\
4 \\
1\end{array}$ & $\begin{array}{l}812.09 \\
811.69 \\
811.43 \\
811.02 \\
810.62\end{array}$ & $\begin{array}{l}123139 \\
123200 \\
123239 \\
123301 \\
123362\end{array}$ & $\begin{array}{l}\left\{\begin{array}{l}123122 \\
123130\end{array}\right. \\
123189 \\
123221 \\
123289 \\
123348\end{array}$ & $\begin{array}{l}2 s^{2} 2 p^{4}{ }^{3} P_{0}-2 s 2 p^{5}{ }^{3} P_{1} \\
2 s^{2} 2 p^{4}{ }^{3} P_{1}-2 s 2 p^{5} P^{3} P_{2} \\
2 s^{2} 2 p^{4}{ }^{3} P_{1}-2 s 2 p^{5} P_{1} \\
2 s^{2} 2 p^{4}{ }^{3} P_{1}-2 s 2 p^{5}{ }^{3} P_{0} \\
2 s^{2} 2 p^{4}{ }^{3} P_{2}-2 s 2 p^{5} P_{2} \\
2 s^{2} 2 p^{4}{ }^{3} P_{2}-2 s 2 p^{5}{ }^{3} P_{1}\end{array}$ & III. Order \\
\hline $\begin{array}{l}3 \\
1 \\
1 \\
3 \\
1 \\
0 \\
1 \\
2 \\
0 \\
1 \\
2 \\
0 \\
1 \\
1\end{array}$ & $\begin{array}{c}792.92 \\
792.50 \\
792.20 \\
791.93 \\
791.48 \\
770.70 \\
770.28 \\
769.39 \\
\overline{756.7} \\
755.8 \\
\overline{-} \\
749.3 \\
748.4\end{array}$ & $\begin{array}{c}126346 \\
126274 \\
126231 \\
126183 \\
126116 \\
129752 \\
129823 \\
129973 \\
- \\
132152 \\
132310 \\
133457 \\
133618\end{array}$ & $\sim 133000$ & $\begin{array}{c}\{71 \\
\{150 \\
\{158 \\
2 p^{3} P_{0,1,2}-\left({ }^{2} P\right) 4 s^{3} P_{0,1,2} ?\end{array}$ & II. Order \\
\hline
\end{tabular}

In front of the slit, there was a small shutter which was closed before an exposure, while the discharge tubes were run at the heaviest possible currents until the last traces of visible bands and of the hydrogen lines disappeared. Table I gives the observed lines which we can attribute with safety to the spectrum of the oxygen atom. The exposures were made with times between 15 minutes and three hours. Most of the lines were measured in the second order against some lines of impurities: O II, NI, N II, CI, CII which have 
been determined by Bowen and Ingram ${ }^{2}$ with great accuracy, and which occurred weakly in our discharge. A few weaker lines have been measured only in the first order against O II, O I, Ne, He. As the intensities vary considerably with the source, intensity values are only relative and only given for lines which belong to one group.

\section{Thi: Excitation Potential of the Ultraviolet (Oxygen Lines}

In order to obtain a classification of the oxygen lines, we investigated the spectrum under varied conditions. A few pictures were taken with the above mentioned positive column in the mixtures: $\mathrm{He}-()_{2}, \mathrm{Ne}-\mathrm{O}_{2}$ and $\mathrm{A}-\mathrm{O}_{2}$. The pressure of the rare gas was not measured but was always adjusted to give the minimum potential drop across the tube. The amount of oxygen added continuously was so small that the stronger lines were just visible in a spectroscope of considerable dispersion. The excitation phenomena of mixtures of rare gases with other gases and vapors have been studied much and discussed in the last years. Although there are some discrepancies between the results of different observers, we can state with safety that the maximum amount of energy available in such a discharge is given by the ionization potential of the rare gas, when the amount of the other component of the mixture is small. These investigations differ only as to whether this amount is transferred to neutral atoms, to metastable atoms or to ions of the second component. In the case of discharges through mixtures of rare gases and molecular gases, the process is complicated by dissociation. By looking over various experiments carried on in this field, one gains the impression that the assumptions of different kinds of collisions are made ad hoc in order to obtain agreement with the observed facts. ${ }^{3}$ Therefore, because the prediction of the excitation limit of unclassified lines seemed to be rather uncertain, we have made no special assumptions as to the processes involved, but we have empirically calibrated our results by the well-known excitation energy of the already classified oxygen lines. Table II gives the results so obtained. The fifth column contains the excitation energy of the upper level of each line in frequency units, the second, third and fourth column the intensities in $\mathrm{A}-\mathrm{O}_{2}, \mathrm{Ne}-\mathrm{O}_{2}$ and $\mathrm{He}-\mathrm{O}_{2}$. Fig. 1 illustrates these results. The exposures are so chosen, that the resonance lines appear on all spectrograms with nearly the same intensity. In argon, only those lines whose upper level is not higher than about 100,000 frequency units above the ${ }^{3} P_{2}$ level are excited with considerable intensity $(>1)$. This photograph shows clearly that 1152 is the lowest excited line of the singlet system because the other lines are either very weak or absent in argon-oxygen. Neon has a very remarkable effect on the intensities. Table II shows that in neon, the line 935.15, whose upper level is about 122800 above the ${ }^{3} P$ term, is strongly

I. S. Bowen and S. B. Ingram, Phys. Rev. 28, 444 (1926).

${ }^{3}$ For a discussion of the various kinds of collision which are necessary to explain the observed facts in mixtures of rare gases and molecular gases, see O. S. Duffendack and H. L. Smith, Phys. Rev. 34, 68 (1929) and O. S. Duffendack and R. A. Wolfe, Phys. Rev. 34, 409 (1929). 

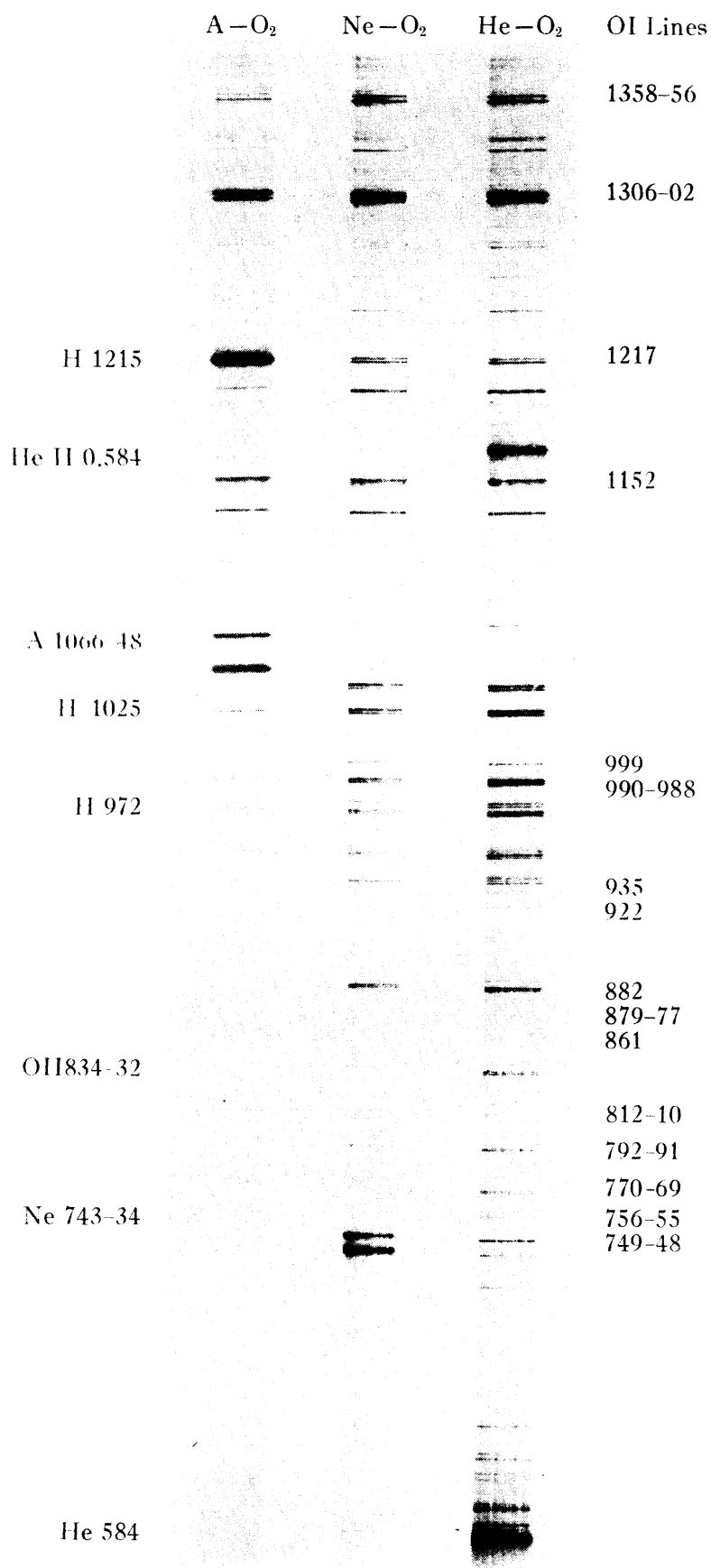

Fig. 1. The ultraviolet $\mathrm{OI}$ spectrum in $\mathrm{A}-\mathrm{O}_{2}, \mathrm{Ne}-\mathrm{O}_{2}$ and $\mathrm{He}-\mathrm{O}_{2}$. 
excited. This phenomenon of increased intensities of certain lines in such discharges often has been mentioned. ${ }^{4}$ It occurs always when either the ionization energy or the energy of the metastable state of the rare gas is approximately equal to the excitation energy of the line of the other component.

TABLE II. Intensities of the ultraviolet O I. Lines under different conditions.

\begin{tabular}{|c|c|c|c|c|}
\hline Wave-length & $\mathrm{A}-\mathrm{O}_{2}$ & $\begin{array}{l}\text { Intensity } \\
\mathrm{Ne}-\mathrm{O}_{2}\end{array}$ & $\mathrm{He}-\mathrm{O}_{2}$ & $\begin{array}{l}\text { Excitation energy } \\
\text { frequency units }\end{array}$ \\
\hline $1358-56$ & 4 & 6 & 6 & 73760 \\
\hline $1306-02$ & 10 & 10 & 10 & 76788 \\
\hline 1217 & - & 3 & 3 & 115922 \\
\hline 1152 & 3 & 4 & 5 & 102670 \\
\hline $1041-39$ & 1 & 4 & 5 & 95222 \\
\hline $1028-25$ & 1 & 4 & 5 & 97481 \\
\hline 999 & - & 4 & 5 & 115922 \\
\hline $990-988$ & 1 & 5 & 8 & 101147 \\
\hline $978-76$ & - & 2 & 3 & 102406 \\
\hline $973-71$ & - & 2 & 3 & 102906 \\
\hline 935 & - & 4 & 3 & 122803 \\
\hline 922 & - & - & 1 & 124326 \\
\hline 882 & - & $\ldots$ & 2 & 129134 \\
\hline $879-77$ & - & 4 & 4 & 113914 \\
\hline 861 & - & - & 1 & 131927 \\
\hline 850 & - & - & 0 & 133413 \\
\hline $812-810$ & - & 1 & 2 & 123348 \\
\hline $792-791$ & - & 2 & 3 & 126231 \\
\hline $770-769$ & - & 1 & 2 & \\
\hline $756-55$ & - & 0 & 1 & \\
\hline $749-48$ & - & - & 0 & 133618 \\
\hline
\end{tabular}

These lines are strongly excited in helium-oxygen but on the other hand, some more lines can be observed in helium which are either weak or absent in neon.

A few words may be said about the excitation of the oxygen arc spectrum in other sources. The positive column in pure oxygen also shows these lines but the intensity at the same amount of current passing through the tube is much smaller than in the mixtures of rare gases and oxygen. In pure oxygen the lines show the usual decrease in intensity with increasing excitation.

The highest amount of excitation with direct current is obtained in the hollow cathode in pure oxygen. In the hollow cathode, all the lines of of O II which have been classified by Bowen ${ }^{5}$ are strongly developed. We also observed the hollow cathode discharge in mixtures of He-O and Ne-O. The spectra so obtained show generally the same lines as the spectra taken with the positive column. The intensity is much smaller and the spectra cannot be so brilliantly excited as in the positive column with great current density.

${ }^{4}$ H. Beutler and B. Josephy, Naturwiss. 15, 540 (1927); F, Paschen, Berl. Ber. p. 536. (1928); Y. Takahashi, Ann. d. Physik 3, 25 (1929); R. A. Sawyer and R. J. Lang, Phys. Rev, 34, 712 (1929).

5 I. S. Bowen, Phys. Rev. 29, 38 (1927). 


\section{The Classification of the New Oxygen Lines}

A discussion of the structure of the oxygen arc spectrum has been given in the above mentioned paper. ${ }^{6}$ It was also shown in this paper that the approximate position of the new terms can be obtained by displacing the RungePaschen terms by the distance from the limit ${ }^{4} S$ to the limit ${ }^{2} D$ and ${ }^{2} P$ respectively. These approximate positions are marked by the horizontal dotted lines in the energy diagram Fig. 2. Almost all of the terms of these two other sets are located quite high above the ordinary ionization limit and the corresponding lines will therefore be weak. The lowest term of the oxygen arc spectrum is built by the addition of a $2 p$-electron to the core $2 s^{2} 2 p^{3}$. It combines therefore with the terms of the configurations $2 s^{2} 2 p^{3} n s$ and $2 s^{2} 2 p^{3} n d$. The lowest terms of these groups, besides the Runge-Paschen terms, are the terms $\left({ }^{2} D\right) 3 s^{3} D, \quad\left({ }^{2} D\right) 3 s^{1} D, \quad\left({ }^{2} P\right) 3 s^{3} P$ and $\left({ }^{2} P\right) 3 s^{1} P$. The position of the triplet terms is already given in the previous paper. Table I shows that the strong diffuse triplet $990-988 \mathrm{~A}$ is resolved in the second order into its six components. The other triplet term $\left({ }^{2} P\right) 3 s^{3} P$ was located in the

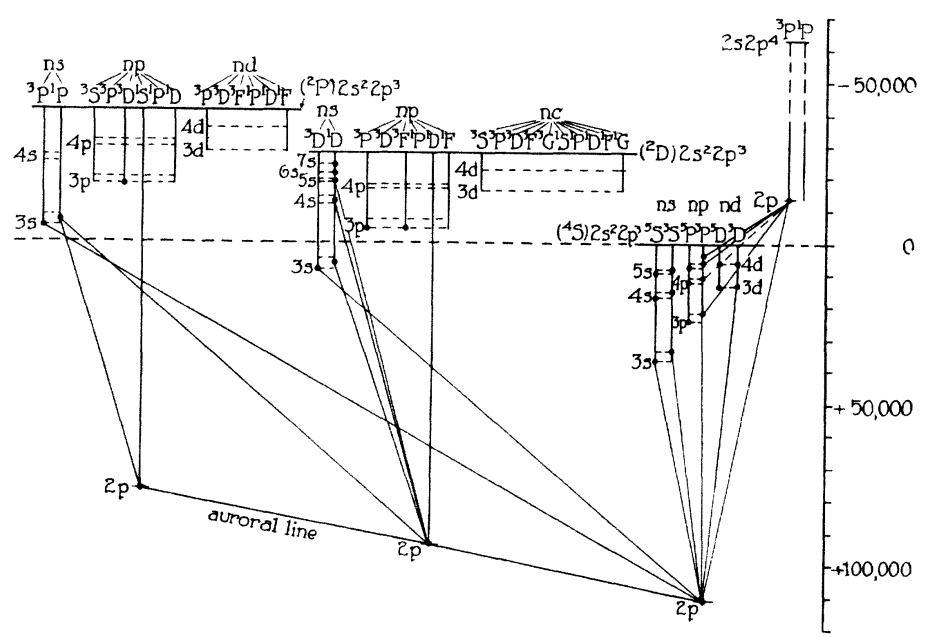

Fig. 2. The term diagram of the OI spectrum.

former paper by a strong combination with the term $\left({ }^{4} S\right) 3 p^{3} P$. Table I gives the combination with the lowest term, $2 p^{3} P$ a strong diffuse triplet at 879-877A. In the second order of the grating, it was impossible to resolve this ${ }^{3} P^{3} P^{\prime}$ group in its six components. This triplet is very strong in the discharge through neon and oxygen.

As Hopfield ${ }^{7}$ has shown the frequency difference of the two strong singlet lines 1217.60 and 999.47 is exactly equal to the frequency of the green auroral line $=17925$ frequency units. We showed in the former paper that these lines are probably the combinations $2 p^{1} S_{0}-\left({ }^{2} P\right) 3 s^{1} P_{1}$ and $2 p^{1} D_{2}-\left({ }^{2} P\right)$ $3 s^{1} P_{1}$ and that a third strong line at $1152 \mathrm{~A}$ is the combination $2 p^{1} D_{2}-\left({ }^{2} D\right)$

${ }^{6}$ R. Frerichs, Phys. Rev. 34, 1239 (1929).

${ }^{7}$ J. J. Hopfield, Phys. Rev. 29, 923 (1927). 
$3 s^{1} D_{2}$. Our experiments on the excitation of the different lines (Table II) show that this last line has a lower excitation potential than the other two, in accordance with this classification.

We have now succeeded in finding five members of the series: $2 p^{1} D_{2}-$ $\left({ }^{2} D\right) n s^{1} D_{2}$. According to Table III, their frequency differences are always between the corresponding frequency differences of the $\left({ }^{4} S\right) n s^{5} S_{2}$ and $\left({ }^{4} S\right)$ $n s^{3} S$ terms. This demonstrates again how accurately the new terms can be obtained by the above mentioned displacement of the Runge-Paschen terms. We have used these five members to calculate the limit of this series in order to locate the important $2 p^{1} D_{2}$ term.

These $\left({ }^{2} D\right) n s^{1} D_{2}$ terms have for their limit the low metastable term ${ }^{2} D$ of the O II spectrum. This term has been located by Bowen ${ }^{8}$ not only by ultraviolet combinations with higher terms but also more accurately by the forbidden combinations with the lowest term: ${ }^{4} S$, i.e. the nebulium lines 3729.91 and 3727.12 , according to his well-known theory. As Hund has shown in Table 34 of his book, the series of the ${ }^{1} D_{2}$ terms have in this case the ${ }^{2} D_{2 \frac{1}{2}}$ component of the doublet term for a limit. This term is inverted and according to Shenstone, ${ }^{9}$ there are some deviations known from Hund's rule in the case of inverted terms. But Shenstone states in his second paper that, in the case of the addition of an $s$-electron, this rule holds even for inverted terms. The possible error caused by a failure of Hund's rule would be in this case, ${ }^{2} D_{2 \frac{1}{2}}-{ }^{2} D_{\frac{1}{2}}=20$ frequency units.

TABLE III. The OI series $2 p^{1} D_{2}-\left({ }^{2} D\right) m s^{1} D_{2}$.

\begin{tabular}{|c|c|c|c|c|c|c|c|}
\hline \multicolumn{2}{|l|}{$\begin{array}{l}\left({ }^{4} S\right) n s^{3} S_{1}: \\
\Delta\left({ }^{4} S\right) n s^{3} S_{1}\end{array}$} & \multicolumn{3}{|c|}{$\begin{array}{ccc}33043.3 & 13612.5 & 7425.6 \\
19430.8 & 6186.9 \\
\end{array}$} & $\begin{array}{c}4672.8 \\
2752.8\end{array}$ & $\begin{array}{r}3210.2 \\
1462.6\end{array}$ & $869.3^{2340.9}$ \\
\hline $2 p^{1} D_{2}-\left({ }^{2} D\right) n s^{1} D_{2}$ & $\left\{\begin{array}{l}\lambda \\
\nu \\
\Delta \nu\end{array}\right.$ & $\begin{array}{l}1152.06 \\
86801 \\
20134\end{array}$ & $\begin{aligned} & 935.15 \\
& 4^{106935}\end{aligned}$ & $\begin{array}{l}\quad 882.88 \\
113266 \\
6331\end{array}$ & $\begin{array}{l}861.63 \\
116059 \\
2793\end{array}$ & $\begin{array}{l}850.7 \\
117545 \\
1486\end{array}$ & \\
\hline $\begin{array}{r}\Delta\left({ }^{4} S\right) n s^{5} S_{2}: \\
\quad\left({ }^{4} S\right) n s^{5} S_{2}:\end{array}$ & & $36069.0^{21710}$ & $\begin{array}{l}0.5 \\
14358.5\end{array}$ & $\begin{array}{l}6637.7 \\
\quad 7720.8\end{array}$ & $\begin{array}{r}2902.9 \\
4817.9\end{array}$ & $\begin{array}{c}1526.0 \\
3291.9\end{array}$ & $\begin{array}{l}900.3 \\
9 \quad 2391.6\end{array}$ \\
\hline
\end{tabular}

We have tried to get higher series members under conditions where the first four members are strong, but the fifth is always weak and it was not possible to obtain higher members. This is not astonishing when one considers how highly unstable such an atom is in a state 24,000 frequency units above the ordinary ionization limit. However, we can estimate the accuracy of this calculation in the following way. The value for the terms $\left({ }^{4} S\right) n s^{3} S_{1}$ and $\left({ }^{4} S\right) 3 s^{5} S_{2}$ which is obtained by applying the Rydberg formula to four series members departs only about 6 frequency units from the value which can be obtained by using eight or nine series members. Adding the fifth member reduces this error to 3 frequency units. We believe, therefore, that the error in the limit of our singlet series caused by this method of calculation is not greater than about 3 frequency units, because these terms are located

${ }^{8}$ I. S. Bowen, reference 5.

${ }^{\ominus}$ A. G. Shenstone, Nature 121, 619 (1928); Nature 122, 727 (1928). 
exactly between the corresponding $\left({ }^{4} S\right) n s^{3} S_{1}$ and $\left({ }^{4} S\right) n s^{5} S_{2}$ terms. The only important source of error will therefore be the comparatively small accuracy in the frequencies at these short wave-lengths.

By this determination the energy values of the terms involved in the emission of the green auroral line are fixed. As we have mentioned above, the classification of this line has been suggested some time ago by McLennan. In the meantime, many attempts have been made to locate these terms. Some predictions of their position have been made by assuming that the relative position of the metastable low terms ${ }^{1} S_{0}$ and ${ }^{1} D_{2}$ is the same for similar spectra. Sommer as well as Kaplan ${ }^{10}$ calculate from the known position of the ${ }^{1} S_{0},{ }^{1} D_{2}$ and ${ }^{3} P$ term in O III the distance ${ }^{1} D_{2}-{ }^{3} P$ to be about 15500 frequency units. This is apparently in very good agreement with the observed position although Sommer as well as Kaplan could not give any theoretical reason for their assumption. In a letter to Nature, ${ }^{11} \mathrm{~J}$. C. McLennan and M. F. Crawford find that the ratio ${ }^{1} D_{2}-{ }^{3} P:{ }^{1} S_{0}-{ }^{1} D_{2}$ is constant and equal to 0.585 for selenium and tellurium and they assume that this ratio is the same for sulphur and oxygen. Thus, by using the value ${ }^{1} S_{0}-{ }^{1} D_{2}=17925$, they find ${ }^{1} D_{2}-$ ${ }^{3} P=10490$. But by comparing the relative positions of such terms in other columns of the periodic table: i.e., C I, Si I, Ge I, Sn I, Pb I or O II, $\mathrm{S}$ II, one finds that the ratio between the low terms in all the cases where they are known, is never constant and, therefore, it seems to be without any justification to predict unknown terms in this way.

Another method of prediction of the approximate position of this ${ }^{1} D_{2}$ term is based on the calculation of the dissociation energy by means of the convergence point of the oxygen bands. Herzberg ${ }^{12}$ has shown that the convergence limit of the Birge-Sponer bands does not correspond to a dissociation into two oxygen atoms in the normal ${ }^{3} P_{2}$ state, but to a dissociation in to one atom in the ${ }^{3} P_{2}$ state and another atom in the ${ }^{1} D_{2}$ state. By comparison of the so calculated dissociation energy $\mathrm{O}_{2} \rightarrow \mathrm{O}_{1 D_{2}}+\mathrm{O}_{3 P_{2}}$ with the chemically measured value $\mathrm{O}_{2} \rightarrow \mathrm{O}_{3 P_{2}}+\mathrm{O}_{3 P_{2}}$, one obtains the value $\mathrm{O}_{1 D_{2}}-\mathrm{O}_{3} P_{2}$. In this way Mecke ${ }^{13}$ obtains ${ }^{1} D_{2}-{ }^{3} P_{2}=1.4$ volts, Henri: $:^{14}{ }^{1} D_{2}-{ }^{1} P_{2}=1.7$ volts the latter value being in better agreement with the observed value: 1.95 volts.

A few words may be said about some other combinations which can be calculated from the known position of the singlet terms. We have not found any intercombination between singlet and triplet terms. The theory of the intensity of intercombinations between singlet and triplet terms has been developed by Houston ${ }^{15}$ for atoms with two outer electrons. This theory predicts, in good agreement with the observations, that intercombinations are strong only when the interval between singlet and triplet terms is small compared with the intervals between the different components of the triplet

${ }^{10}$ L. A. Sommer, Zeits. f. Physik 51, 451 (1928); J. Kaplan, Phys. Rev. 33, 638 (1929).

" J. C. McLennan and M. F. Crawford, Nature 124, 874 (1929).

${ }^{12}$ G. Herzberg, Zeits. f. Physik Chem. 4, 13, 233 (1929).

${ }^{13}$ R. Mecke, Naturwiss. 17, 996 (1929).

${ }^{14}$ V. Henri, Nature 125, 201 (1930).

${ }^{15}$ W. V. Houston, Phys. Rev. 33, 297 (1929). 
TaBle IV. Term table of the OI spectrum.

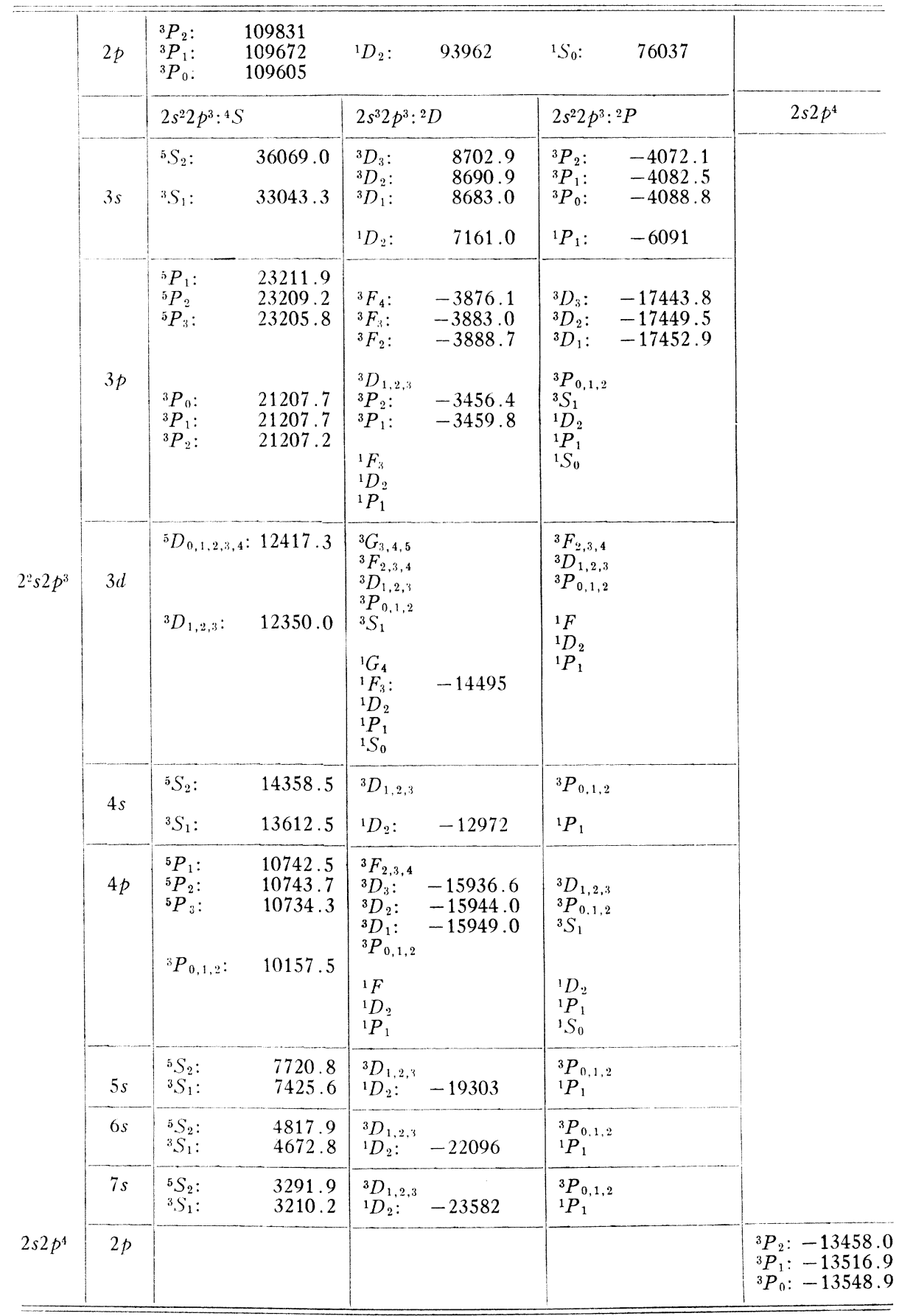




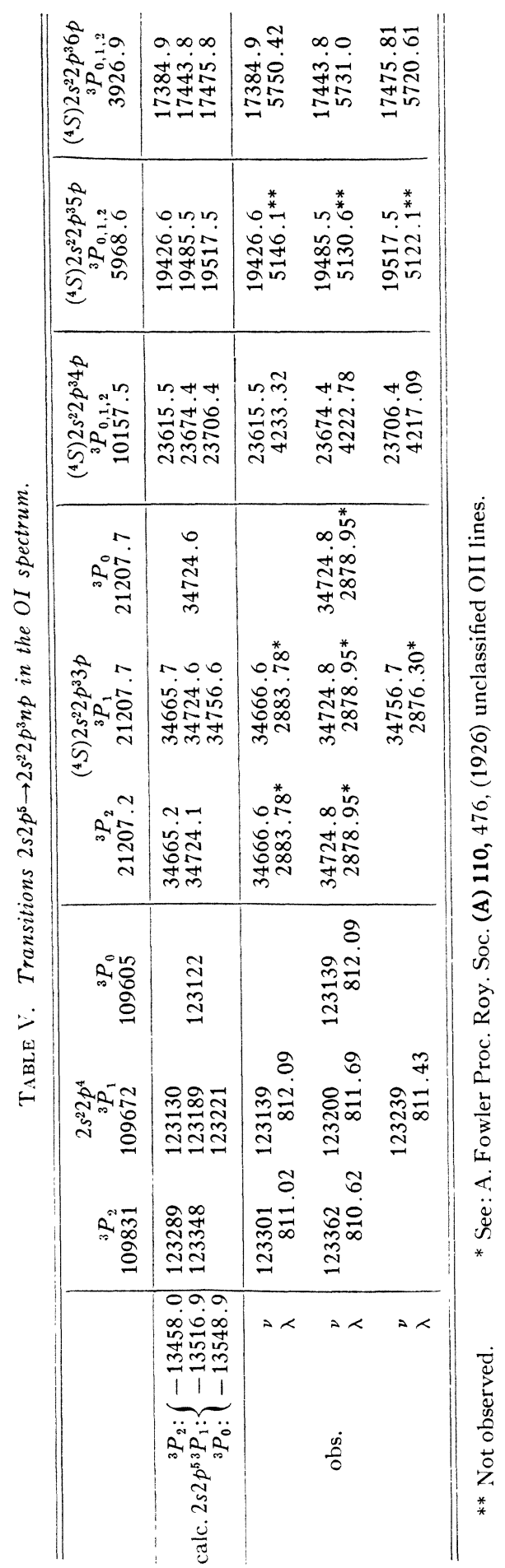


term. Although this theory cannot be applied to the more complicated case of the oxygen atom, the very small intervals of the oxygen terms may be responsible for the missing intercombination lines. It is in agreement with Houston's theory that, in the case of selenium and tellurium, where the intervals are much larger, such intercombinations have been found by $\mathrm{Mc}$ Lennan.

The green auroral line is according to its classification, the exact analogue of the nebular line $4363.21 \mathrm{~A}$. We should therefore expect to find also the other combinations ${ }^{3} P_{2}-{ }^{1} D_{2}$ and ${ }^{3} P_{1}-{ }^{1} D_{2}$ which correspond to the nebular lines 5006.84 and 4958.91A. According to Table IV, these lines are located at 6299 and $6363 \mathrm{~A}$ with an error of approximately $\pm 5 \mathrm{~A}$. These lines are neither found in the laboratory nor in the spectrum of the aurora or of the night sky.

Among the new triplet terms (Table IV), the term: $2 s 2 p^{5}{ }^{3} P$ is especially interesting. By application of the irregular doublet law to the oxygen-like isoelectronic spectra, Mack and Sawyer ${ }^{16}$ have predicted its position as about 15000 frequency units above the ordinary ionization limit, the ${ }^{4} S$ term of the ion, in good agreement with the observed value. Under the unclassified lines in the near ultraviolet and in the visible, there are some triplets which give the intervals 58.9 and 32 frequency units. Table $\mathrm{V}$ shows that these triplets are combinations of this $2 s 2 p^{53} P$ term with the terms: $\left({ }^{4} S\right) 2 s^{2} 2 p^{3}$ $n p^{3} P$. The combination $\left({ }^{4} S\right) 2 s^{2} 2 p^{3} 5 p^{3} P-2 s^{2} p^{5}{ }^{3} P$ is not known, perhaps due to the small sensitivity of the photographic plates in this region. The term: $2 s 2 p^{5}{ }^{3} P$ combines with a ${ }^{3} P$ term at 3926 and the application of the Rydberg formula shows that this term is the hitherto unknown term $\left({ }^{4} S\right) 2 s^{2} 2 p^{3} 6 p^{3} P$.

We are indebted to the Rockefeller Foundation for the grant of a fellowship, to Professor R. A. Millikan for extending the facilities of the Norman Bridge Laboratory and to Professor I. S. Bowen for helpful discussions and suggestions.

Note added with proof: Prof. Paschen writes me that he has recently observed the two lines ${ }^{3} P_{2}-{ }^{1} D_{2}$ and ${ }^{3} P_{1}-{ }^{1} D_{2}$ at 6300.06 and $6363.86 \mathrm{~A}$ respectively in a discharge through pure oxygen. This proves that the above given calculation of the singlet terms is accurate within 0.5 frequency units. S. Goudsmit, Phys. Rev. 35, 1325, (1930) finds by theoretical calculations the above mentioned value of McLennan in disagreement $w$ ith our measurements. V. Kondratjew, Zeits. f. Phys. Chem. B. 7, 70, (1930) obtains from the oxygen bandspectra the value 1.91 volts for the difference ${ }^{3} P_{2}-{ }^{1} D_{2}$ in very good agreement with the observed value 1.95 volts.

16. J. E. Mack and R. A. Saw yer, Phys. Rev. 35, 299 (1930). 


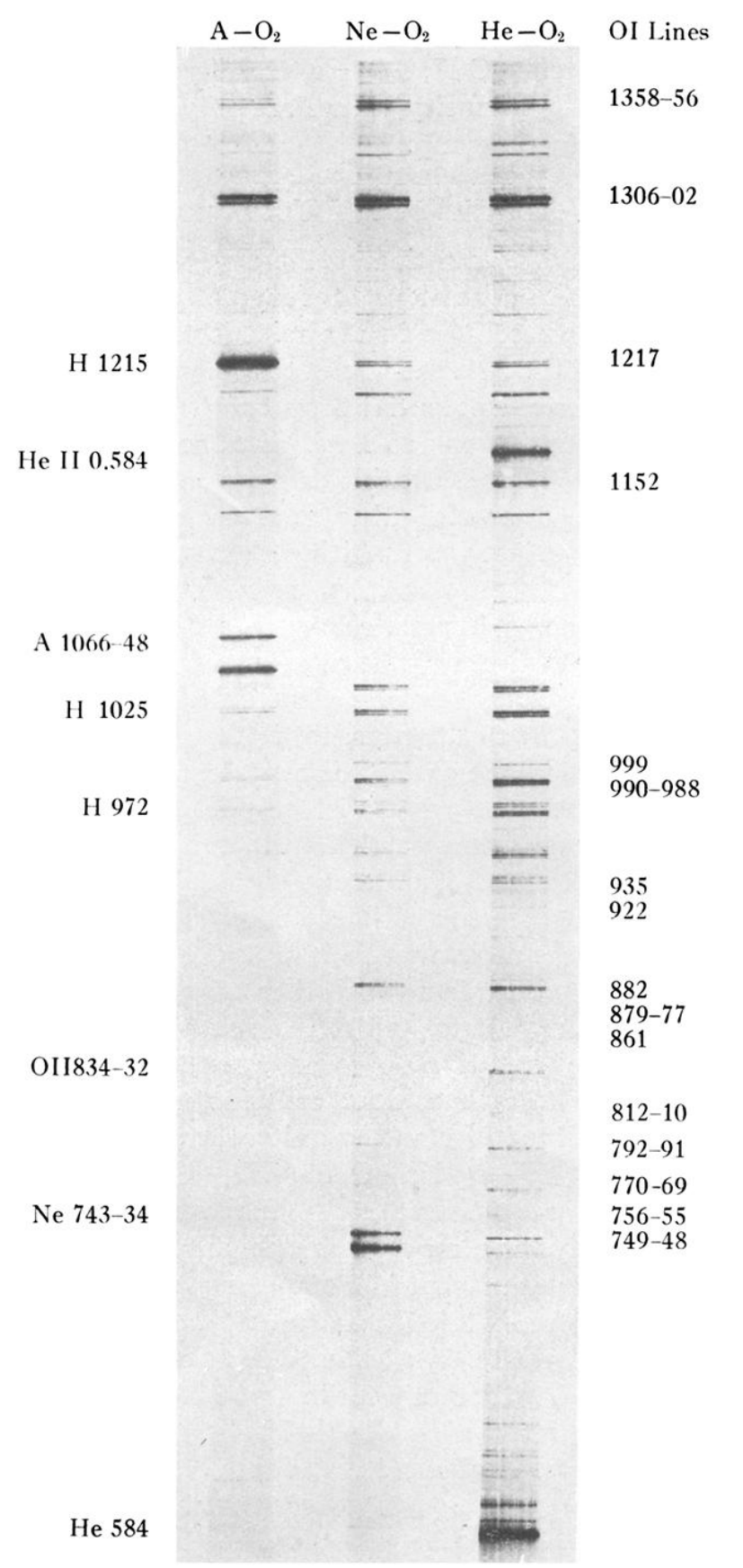

Fig. 1. The ultraviolet $\mathrm{OI}$ spectrum in $\mathrm{A}-\mathrm{O}_{2}, \mathrm{Ne}-\mathrm{O}_{2}$ and $\mathrm{He}-\mathrm{O}_{2}$. 